\title{
Research Plan of Integrated Music Activities of Gregarious Elderly
}

\section{People}

\author{
Defang Qu ${ }^{1}$
}

${ }^{1}$ Xi'an University, Xi'an, 710065

\section{KEYWORDS: Integrated Music Activities; Gregarious Elderly; Research Plan}

\begin{abstract}
Aging social trends now unstoppable, pension issues pushed to the center of the social scene. In efforts to promote the social old-age infrastructure, intelligent building services, but also the "cultural care", "spiritual endowment" of the building direction referred to the important agenda. Musical event is an important recreational activity, the "spiritual care" act as an important role. Music event in the end is how to play a role in "spiritual care" in? How to better play the role? Elderly mental activity to clarify the law when participating in musical activities is the key to finding the answer.
\end{abstract}

\section{Introduction}

Music positive meaning of life, is an indisputable fact that psychology of music through a variety of scientific methods and means, which carried out extensive and in-depth research. The reaction not only the law of the living body to stimulate the music made a description of the music but also fully confirmed the human body can produce physical and psychological authenticity positive effects. A set of elderly living in nursing homes designed to fit their music program of activities is to take advantage of musical activities, and to exert a positive effect on the life of music unique to improve quality of life in elderly individuals. Research Geriatric Mental musicology oriented research has laid a solid theoretical foundation. While the older music education, music therapy and senile elderly community-based musical entertainment development issues provided valuable operational experience. In the old university, community arts training institutions, community elderly activity centers and other places have opened a wide range of older music courses. Long-term teaching practice led to a large number of theoretical achievements music teaching methods and materials for the elderly and other music education. Such as "elderly piano based tutorial" elderly Piano Tour ", teach the elderly learn to sing," Passion Square Community Chorus: learning to teach the elderly chorus "Dance Tutorial old university textbooks, etc., as well as a large number of research literature, audio and video material. aged for all types of music education activities in the community to carry out extensive and in the end of life of the elderly have many positive? our study reveals a series of old age began to learn music on delaying the elderly cognitive aging process, maintain or enhance the basic cognitive abilities of older persons, both positive and reliable effect. studies have shown that older people learning music not only has a positive role in the development of intelligence, and, learning music than non-elderly group learning music group the elderly have higher levels of subjective well-being of the family practice and theory in old music education, the subject of this study provides a wealth of operational experience and theoretical material.

Community, squares, parks and other places initiated colorful musical entertainment. Various choir, dance team, the people band, drama group, mostly organized group rehearsals, performances, competitions activities; down in a tree, gazebo, pond, three thousand three hundred twenty-two 
random combination of free performances everywhere visible. In recent years, the prevalence of square dance, with Chinese characteristics has become the cultural landscape. Major news media after another appeared positive and negative reports on the Square music. The prosperity of the masses of music culture theory researchers has become the focus of attention on the music community, plaza music culture research articles and papers diversified emerging. These colorful musical entertainment and related theoretical research, problem-oriented research also provides operating practical experience and theoretical support.

\section{Elderly Integrated Music Activities in the Field of Psychology}

Actors mainly refers to actors objective fact itself has different from others, the more stable the physical and psychological characteristics of the facts, including appearance including signs, health, personality characteristics, psychological experience and so on. The above aspects are actors that have relatively stable individual characteristics, they interact together to form a long-term psychological impact on the body farm "quasi-facts" is formed, every aspect of these objective facts they have played their different roles.

This refers to the individual appearance and signs a complete external physiological shape, including height and weight of the individual physique, body proportion, face shape, facial proportions, color and so on. The value of human existence is the appearance of individual signs of life in the material level of the most primitive expression. In the process of performing music, "body image" is the impact of "quasi-facts" actors psychological field formed appearance signs. Body image is divided into "positive body image" and "negative body image." The former formation of individual personality will play a positive role in promoting the individual's self-confidence will improve and increase individual participation in group activities of interest and enthusiasm; contrast, the "negative body image" would be the formation of a negative impact of individual personality, will hinder the formation of individual self-confidence, so that individual participation in group activities, because of their "negative body image" inferiority complex, and psychological burden that evade or resist group activities. Aging skin and bone deformation, so that the elderly population looks young compared to the conditions and signs, significant degradation, so that in daily life, self body image concern and attention is also much lower than the young. Under visual focus, although not dance music events as entirely body language to show, but it is after all the performing arts, and therefore, in the context of music, performing groups in the body, its external image, and in everyday life as compared with more significance. So, yes, the elderly participate in music activities, based on the "positive body image" and "negative body image" of two distinct psychological state and it will produce different effects its formation Performance Psychology Field.

To the health of elderly people, because the body physiological function of aging, various geriatric diseases have emerged, resulting in a reduced ability of individual behavior. One study showed that: "The United States more than $80 \%$ of the elderly suffer from at least one chronic disease, $50 \%$ of elderly people suffer from at least two chronic diseases." This causes almost all the elderly in their own psychological and behavioral health concerns ability not confident. So, when the elderly engaged in musical activities, the health condition of the individual, it would affect their participation in musical activities important facts conditions and psychological factors. However, "with age, individual differences in sensory and motor function gradually emerged sensory and motor function some of the sharp decline in the elderly; while other sensory and motor function of the elderly, but there was little change." Not only music events each participating state of health of the elderly there are individual differences, and their own health psychological assessment also 
determined that there are individual differences. "The results show that the negative stereotypes of aging function to play the elderly and bring pressure to disrupt;. A positive impression of the elderly is able to reduce stress and promote their capabilities" Therefore, due to individual differences in personality and mental endurance different from the same disease, different individuals will have different psychological effects. Because the elderly have occurred sudden onset of death or when participating in musical activities case, then, had any serious illness or sudden death in the face life experience of older individuals show than not have the same experience of the individual, more worried about their own health situation. Therefore, the elderly are engaged in musical activities, not only need the support of a certain physical strength and energy, intricate rhythm, pitch, intensity, timbre changes, but also the performers clear mind and fine motor dexterity to complete. These characteristics and requirements is bound to the health problems of elderly individuals bring some psychological pressure.

\section{Definition of Research Questions}

The end result of the research is to operate through repeated experiments, summed up some music for the practical operation methods appropriate settlements pension institutions for the elderly. These possible to borrow the practical operation of the method, the music properly integrated into the social life in the elderly pension institutions, the positive effects of music to play for life, in order to achieve enriching the lives of the elderly, improve mood, play health care, longevity, enhance the role of quality of life. The purpose of this article is to test the operation-oriented design and implementation of specific programs of music events next task will be carried out, to make a research idea, to do the preparatory work for the application of the theory.

The title of this article "Elderly Integrated Music Activities gregarious manner of social research idea is an old one concentrated elderly living in institutions, nursing homes, homes for the elderly and other professional pension institutions. These institutions concentrated elderly population live, generally have a certain amount of scale , professional management of pension institutions, their daily diet, healthy, recreational activities have a uniform arrangement\%. comprehensive music activity is different from that arts training institutions, universities for the elderly opened by professional music teaching activities or drama classes, choir , dance team and other groups musical activities and professional "music therapy carried out acts against the elderly. But rather for social institutions for the elderly pension number uncertain, blow, pull, play, sing, dance, and visual appreciation and other eclectic integrated music events.

\section{Research Methods}

This paper is mainly used for the study of the following methods: first, the theoretical speculation method. In determining research topics when the significance of the research, positioning research questions, study design and analysis of the results of the study and summarize other aspects, mainly the use of the theory of speculative research methods and materials of the existing facts, analyze and summarize the conclusions. Even in the implementation of established research programs, when the specific contents of operation and control, are inseparable from the theory of speculative participation.

Study of individual information on older subjects, in addition to some external indicators can be obtained through observation, measurement obtained outside the individual's inherent understanding of the issues, attitudes, needs, desires and feelings result of intuition, etc., only the use of the survey method to obtain individual direct expression and confirmation in order to obtain a 
more accurate and reliable factual information. Research tasks and findings of this subject precisely mainly located in the older inner spiritual dimension.

Third, Its literature. This is a very specific methodology vitality, which can be obtained from the limitations of time and space-related information. Ancient and modern literature we can see that many researchers can not be experienced, witnessed things and phenomena. Especially in today's technological advances, the literature only presents a large number of data and text messages, as well as high-definition pictures and information on the impact of the scene can be effectively record of the incident. Similarly, during the study of this subject, for research to grasp the current situation, the topic for the determination of significance of the topic for understanding the design of the research process, must master the large number of documents on the basis of the analysis in order to make judgment. Even for research institutions and the implementation of experimental operation of the choice of venue, we need to provide some literature as a reference to the decision. In particular, the research across psychology, gerontology, music, sociology, clinical medicine, rehabilitation medicine, etc., for learning academic knowledge, operating experience for reference, the vast majority must be obtained through literature.

\section{Conclusion}

This article is to study theoretical assumption made, the principles to be followed in the study and analysis of the positioning of the research methods to be employed in the introduction, the study analyzes problems foreseen. Not the study of specific embodiments, it can be used to develop specific research programs premise. The next step will be to work on specific research process, research, research design implementation programs.

\section{Acknowledgements}

Project: Xi'an 2015 Annual Social Science Fund Project Planning: " To Solve the Problem of the Elderly Spiritual Needs in Music the Way " Initial Results.

Project No.: 15WL09.

\section{REFERENCE:}

[1] Luo Xiaoping. Geriatric Mental Musicology Discussion [J]. Huang Zhong, 2006 (2): 75-81.

[2] Luo Xiaoping, Ren Jie. Piano Learning Effect In The Process Of Cognitive Aging Research [J]. People's Music, 2009 (8): 73-75.

[3] Ren Jie, Luo Xiaoping. Affect The Piano Study On The Processing Speed Of The Elderly, Working Memory And Fluid Intelligence [J]. Chinese Journal Of Clinical Psychology, 2009 (4): 396-399.

[4] Duan Jianhua. Swb Overview [J]. Psychology, 1996 (4): 46-51.

[5] Liu Rengang. Study Swb And Its Influencing Factors Gong Yaoxian [J]. Chinese Journal of Clinical Psychology, 2000 (2): 73-78. 